\title{
Ternary Permutable Semigroups of the First Kind
}

\author{
Rushadije R. HALILI *, Merita AZEMI , Lazim KAMBERI \\ Faculty of Natural Sciences and Mathematics, University of Tetovo, Ilinden n.n., 1200 Tetovo, Republic of \\ North Macedonia
}

*Corresponding Author: Rushadije R. HALILI, Faculty of Natural Sciences and Mathematics, University of Tetovo, Ilinden n.n., 1200 Tetovo, Republic of North Macedonia

\begin{abstract}
A semigroup $S$ is called a permutable semigroup if $\rho \cdot \sigma=\sigma \cdot \rho$ is satisfied for all congruences $\rho$ and $\sigma$ of $S$.A non empty set $S$ together with a ternary multiplication denoted by juxtaposition, is said to be a ternary semigroup if ( $a b c) d e=a$ ( $b c d$ ) $e=a b$ (cde) for all $a, b, c, d, e \in S$. In this paper we deal with permutable ternary semigroups of the first kind.
\end{abstract}

Keywords: Ternary semigroup, permutable semigroup.

\section{INTRODUCTION}

The first paper about permutable semigroups is [4] where some general theorems are proved and the commutative permutable semigroups are described.

Using the terminology of [5], a semigroup $S$ is called a semigroup of type $A$ if it is a semilattice of a nil semigroup $S_{0}$ and a rectangular group $S_{1}=L \times G \times R$ with $|L| \leq 2,|R| \leq 2$ ( $L$ is a left zero semigroup, $G$ is a group, $R$ is a right zero semigroup).A semigroup $S$ of type $A$ is called of the first kind if $a \in S_{1} a S_{1}$ for every $a \in S$.

Let $S$ be a ternary permutable semigroup of the first kind. Then $S$ is a semilattice of a nil semigroup $S_{0}$ and a rectangular abelian group $S_{1}=L \times G \times R$ with $|L| \leq 2,|R| \leq 2$ ( $L$ is a left zero semigroup, $G$ is a group, $R$ is a right zero semigroup). It is obvious that $S_{1}$ is a rectangular band $L \times R$ of discoint subgroups $G_{i j}=\{i\} \times G \times\{j\}(i \in L, j \in R)$ and the idempotent elements of $S_{1}$ are the identity elements $e_{i j}=(i, e, j)$ of $G_{i j}$ (here $e$ denotes the identity element of $G$ ).

Introduce the following notation: for an element $t$ of a non-empty set $T$ containing at most two elements, let $\bar{t}=t$ if $|T|=1$ and let $\bar{t} \in T-\{t\}$ if $|T|=2$.

Definition1:A semigroup $S$ is called a permutable semigroup if $\rho \cdot \sigma=\sigma \cdot \rho$ is satisfied for all congruences $\rho$ and $\sigma$ of $S$.

Definition2: A non empty set $S$ together with a ternary multiplication denoted by juxtaposition, is said to be a ternary semigroup if $(a b c) d e=a(b c d) e=a b(c d e)$ for all $a, b, c, d, e \in S$.

Definition3: A ternary semigroup $S$ is said to be commutative if $x_{1} x_{2} x_{3}=x_{\sigma(1)} x_{\sigma(2)} x_{\sigma(3)}$ for every permutation $\sigma$ of $\{1,2,3\}$ and $x_{1}, x_{2}, x_{3} \in S$. 


\section{Ternary Permutable Semigroups of THE FirSt Kind}

Lema 1: If $S$ is a ternary permutable semigroup of the first kind then, for every $a \in S, i \in L$ and $j \in R$ we have

(i) $e_{i j} a=e_{i \tilde{j}} a$.

(ii) $a e_{i j}=a e_{i j}$.

Proof. As $S$ is ternary permutable semigroups for every $a \in S, i \in L$ and $j \in R$ we have $e_{i j} a=e_{i j} e_{i \bar{j}} e_{i j} a=e_{i j} e_{i j} e_{i \bar{j}} a=e_{i \bar{j}} a$

and

$a e_{i j}=a e_{i j} e_{i j} e_{i j}=a e_{i j} e_{i j} e_{i j}=a e_{i j}$

Introduce the following notations. For arbitrary $i \in L$ and $j \in R$, let

$A_{i}=e_{i j} S=e_{i \bar{j}} S$ and $B_{j}=S e_{i j}=S e_{i \bar{j}}$.

It is clear that $A_{i}=G_{i j} \cup G_{i \bar{j}} \cup e_{i j} S_{0}$ and $B_{j}=G_{i j} \cup G_{\bar{i} j} \cup S_{0} e_{i j}$.

A semigroup is said to be left (right) commutative if it satisfies the identity $a b c=b a c(a b c=a c b)$.

Lema 2: Let $S$ be a ternary permutable semigroup of the first kind. Then $A_{i}(i \in L)$ and $B_{j}(j \in R)$ are left and right commutative subsemigroups of $S$, respectively.

Proof. It is clear that $e_{i j}$ is left identity elements of $A_{i}$. Then, for arbitrary elements $a, x, y \in A_{i}$, $x y a=e_{i j} x y a=e_{i j} y x a=y x a$.

Hence $A_{i}$ is left commutative. The proof of the assertion for $B_{j}$ is similar.

Lemma 3: Let $S$ be a ternary permutable semigroup of the first kind. Then $S=A_{i} \cup A_{i}=B_{j} \cup B_{\bar{j}}(i \in L, j \in R)$.

Moreover, $A_{i} \cap A_{\bar{i}}$ and $B_{j} \cap B_{\bar{j}}(i \in L, j \in R)$ are ideals of $S$.

Proof. Let $S$ be a ternary permutable semigroup of the first kind. Then for every $a \in S$ there is an element $e_{i j} \in E\left(S_{1}\right)$ such that $a=e_{i j} a \in A_{i}$.

Thus $S=A_{i} \cup A_{i}(i \in L)$. Similarly, $S=B_{j} \cup B_{\bar{j}}(j \in R)$.

It is clear that $A_{i} \cap A_{i} \neq \varnothing$ is a right ideal of $S$. Let $s \in S, a \in A_{i} \cap A_{i}$ be arbitrary elements. Then $e_{t, k} a=a$ for every $t \in L, k \in R$. Assume $s \in A_{i}$. As $A_{i}$ is a subsemigroup of $S, s a \in A_{i}$. As $S$ is of the first kind, $a=a t$ for an element $t \in S_{t}$.

Thus for arbitrary $j \in R, e_{i j} s a=e_{i j} s a t=e_{i j} a s t=a s t=e_{i j} a s t=e_{i j} s a t=s a$, that is $s a \in A_{i}$. 
Thus $s a \in A_{i} \cap A_{i}$. Hence $A_{i} \cap A_{i}$ is an ideal of $A_{i}$. We can similarly prove that $A_{i} \cap A_{i}$ is an ideal of $A_{i}$. Hence $A_{i} \cap A_{i}$ is an ideal of $S$. The proof of the assertion that $B_{j} \cap B_{j}$ is an ideal of $S$ is similar. ㅁ

Lema 4:If $f$ is an idempotent element of a ternary semigroup $S$, then

$\eta_{f}=\{(x, y) \in S \times S \mid f x=f y\}$ and $\mu_{f}=\{(x, y) \in S \times S \mid x f=y f\}$ are congruences on $S$.

Proof. It is clear that $\eta_{f}$ is a right congruence. Let $x, y, z$ be arbitrary elements of $S$ such that $(x, y) \in \eta_{f}$. Then $f z x=f f z x=f z f x=f z f y=f f z y=f z y$ and so $(s x, s y) \in \eta_{f}$.

Hence $\eta_{f}$ is a congruence on $S$. The proof is similar for $\mu_{f}$.

Lema 5: If $S$ is a ternary permutable semigroup of the first kind then, for every $i \in L$ and $j \in R$

(1) $\eta_{e_{i j}}=\eta_{e_{i j}}=\eta_{e_{i j}}=\eta_{e_{i j}}$.

(2) $\mu_{e_{i j}}=\mu_{e_{i j}}=\mu_{e_{i j}}=\mu_{e_{i j}}$

Proof. By lema $1, \quad \eta_{e_{i j}}=\eta_{e_{i j}}$ and $\eta_{e_{i j}}=\eta_{e_{i j}}$.

We show that $\eta_{e_{i j}}=\eta_{e_{i j}}$.

Assume that $(a, b) \in \eta_{e_{i j}}$ for some $a, b \in S$. Then $e_{i j} a=e_{i j} b$ and so

$$
e_{i \tilde{j}} a=e_{i \tilde{j}} e_{i j} a=e_{i \tilde{j}} e_{i j} b=e_{i j} b .
$$

Then $(a, b) \in \eta_{e_{i j}}$. Thus, $\quad \eta_{e_{i j}} \subseteq \eta_{e_{i j}}$. Similarly $(a, b) \in \eta_{e_{i j}}$ for some $a, b \in S$, then $e_{i j} a=e_{i j} b$ and so $e_{i j} a=e_{i j} e_{j i} a=e e_{j j} b=e b_{i}$. Then $(a, b) \in \eta_{e_{i j}}$. Thus $\eta_{e_{i j}} \subseteq \eta_{e_{i j}}$ Hence $\eta_{e_{i j}}=\eta_{e_{i j}}$ ( (1) is satisfied). The proof of (2) is similar.

Lema 6: If $S$ is a ternary permutable semigroup of the first kind then for every $i \in L$ and $j \in R, A_{i} \cong S / \eta$ and $B_{j} \cong S / \mu$.

Proof. Let $[a]_{\eta}$ denote the $\eta$-class of $S$ containing the element $a$ of $S$. We show that $[a]_{\eta}=\left(E\left(S_{1}\right)\right) a$. Assume $(x, y) \in \eta$ for some $x, y \in A_{i}$. As $e_{i j}$ is a left identity element of $A_{i}$, we have $x=e_{i j} x=e_{i j} y=y$. Thus $\eta / A_{i}=i d_{A_{i}}$ where $\eta / A_{i}$ is the restriction of $\eta$ to $A_{i}$ and $i d_{A_{i}}$

Is the identity relation of $A_{i}$. Let $a \in S$ be an arbitrary element. Then by lema3, $S=A_{i} \cup A_{i}$, and so there is an element $i \in L$ such that $a \in A_{i}$. As $e_{i j} a=e_{i j} e_{i j a} j \in R$, we have $\left(a, e_{i j} a\right) \in \eta$.

Thus $[a]_{\eta}=\left\{a, e_{i j} a\right\}$.

Since $a=e_{i j} a=e_{i \bar{j}} a$ and $e_{i \bar{j}} a=e_{\overline{i j}} e_{i j} e_{i \bar{j}} a=e_{i \bar{j}} e_{\overline{i j}} e_{i j} a=e_{i j} a$, we get $[a]_{\eta}=\left\{a, e_{i j} a\right\}=\left(E\left(S_{1}\right)\right) a$.

This result implies that $\left|A_{i} \cap[a]_{\eta}\right|=1$ for every $a \in S$. Let $\Phi_{i}$ denote the mapping of $S / \eta$ to $A_{i}$ defined by $\Phi_{i}:[a]_{\eta} \rightarrow A_{i} \cap[a]_{\eta}$. Then $\Phi_{i}$ is bijective. As $\left(A_{i} \cap[a]_{\eta}\right)\left(A_{i} \cap[b]_{\eta}\right) \in\left(A_{i} \cap[a b]_{\eta}\right)$ we get $\Phi_{i}(a) \Phi_{i}(b)=\left(A_{i} \cap[a]_{\eta}\right)\left(A_{i} \cap[b]_{\eta}\right) \in\left(A_{i} \cap[a b]_{\eta}\right)=\Phi_{i}(a b)$ which means that $\Phi_{i}$ is a homomorphism. 
Thus $\Phi_{i}$ is an isomorphism of $S / \eta$ onto $A_{i}$. The proof of $B_{j} \cong S / \mu$ is similar $\square$.

Corollary 1: Let $S$ be a ternary permutable semigroup of the first kind. Then for every $i \in L$ and $j \in R, \phi_{i}: a \rightarrow a^{\prime}=e_{i j} a\left(a \in A_{i}\right)$ and $\Psi_{j}: b \rightarrow b^{\prime}=b e_{i \bar{j}}\left(b \in B_{j}\right)$ are isomorphisms of $A_{i}$ and $B_{j}$ onto $A_{\bar{i}}$ and $B_{\bar{j}}$, respectively.

\section{REFERENCES}

[1] Cliford A.H. and G.B. Preston, The algebraic theory of semigroups, Math. Surveys N. Amer. Math. Soc. Providence, 1(1961).

[2] Attila Nagy, Medial permutable semigroups of the first kind, Semigroup forum (2008),297-308. [3]Sioson FM. Ideal theory in Ternary semigroups, Math Japonica (1965) 63-84.

[3] Hamilton H., Permutabiliti of congruences on commutative semigroups, Semigroup Forum, 10 (1975) 55-66.

[4] Bonzini, C., Cherubini, Medial permutable semigroups, Coll Math. Soc. Janos Bolyai, vol.39(21- 39)1981.

[5] A. Deak and A. Nagy, Finite permutable Putcha semigroups.

[6] Rushadije R. Halili,D. Ibishi, Exponential permutable semigroups, Journal of advances in Mathematics, vol.9(2014).

Citation: Rushadije R. HALILI, Ternary Permutable Semigroups of the First Kind, International Journal of Scientific and Innovative Mathematical Research (IJSIMR), vol. 8, no. 10, pp. 14-17, 2020. Available : DOI: https://doi.org/ 10.20431/2347-3142.0810002

Copyright: (C) 2020 Authors. This is an open-access article distributed under the terms of the Creative Commons Attribution License, which permits unrestricted use, distribution, and reproduction in any medium, provided the original author and source are credited. 